Canadian University Music Review

Revue de musique des universités canadiennes

\title{
Actes du colloque « Musicologie historique et musicologie théorique : une coexistence est-elle possible ? " : École de musique, Université Laval, Québec, 5 mars 1993
}

\section{Jean-Michel Boulay, Paul Cadrin, Marc Honegger, Jean-Jacques Nattiez et Vivianne Émond}

Numéro 14, 1994

URI : https://id.erudit.org/iderudit/1014316ar

DOI : https://doi.org/10.7202/1014316ar

Aller au sommaire du numéro

Éditeur(s)

Canadian University Music Society / Société de musique des universités

canadiennes

ISSN

0710-0353 (imprimé)

2291-2436 (numérique)

Découvrir la revue

Citer ce compte rendu

Boulay, J.-M., Cadrin, P., Honegger, M., Nattiez, J.-J. \& Émond, V. (1994). Compte rendu de [Actes du colloque «Musicologie historique et musicologie théorique : une coexistence est-elle possible ? " : École de musique, Université Laval, Québec, 5 mars 1993]. Canadian University Music Review / Revue de musique des universités canadiennes, (14), 161-182. https://doi.org/10.7202/1014316ar

All Rights Reserved (C Canadian University Music Society / Société de musique des universités canadiennes, 1994
Ce document est protégé par la loi sur le droit d'auteur. L'utilisation des services d'Érudit (y compris la reproduction) est assujettie à sa politique d'utilisation que vous pouvez consulter en ligne.

https://apropos.erudit.org/fr/usagers/politique-dutilisation/ 
Acide," and Paul F. Rice, "Rameau's Overtures." The School of Music at Memorial University of Newfoundland provided an evening concert of music by Haydn, Mozart and C.P.E. Bach that featured student and faculty performers. The society welcomes this interest in music, and the fine arts in general, and hopes that it will continue to grow and prosper in future meetings. In addition, the society publishes a select number of the conference papers in its journal, Lumen (formerly Man and Nature/L'homme et la nature).

It is with regret that I must report that the twentieth meeting of the society, scheduled to be held at Carleton University in October 1993, will be delayed until 13-16 October 1994, when it will be hosted by the University of Saskatchewan in Saskatoon. Although this delay will disappoint many, it will give the members of the society ample time to plan their papers. To that end, I should like to extend a special invitation to all whose interests lie in the fine and performing arts to consider submitting an abstract for the twentieth meeting of the society. The actual call for papers will be announced at a later time. Those interested in joining the society should contact Dr. Paul Wood, Department of History, University of Victoria, P.O. Box 3045, Victoria, British Columbia, V8W 3 P4.

\section{Actes du colloque « Musicologie historique et musicologie théorique : une coexistence est-elle possible ?" École de musique, Université Laval, Québec 5 mars 1993}

\section{Introduction}

L'École de musique de l'Université Laval a été l'hôte, au cours de l'après-midi du 5 mars 1993, d'un colloque portant sur la coexistence de deux types de musicologies : la musicologie historique et la musicologie théorique. L'organisateur du colloque, Marc-André Roberge, avait invité quatre musicologues représentant les deux tendances à venir présenter une prise de position de manière à lancer le débat entre les invités eux-mêmes et entre les invités et le public. Une bonne trentaine de personnes venant du Québec, de l'Ontario et du Nouveau-Brunswick, et dont plus de la moitié étaient des étudiants de deuxième et troisième cycles en musicologie, se sont réunies à la salle Henri-Gagnon du pavillon Louis-Jacques-Casault de l'Université Laval. 
Dans l'invitation à participer au colloque, qui avait été diffusée au cours de l'automne 1992, on pouvait lire :

La musicologie actuelle compte deux grandes tendances opposées : historique et théorique. La musicologie historique, de tradition humaniste, se consacre entre autres à l'étude de la vie musicale, à la description des sources, à l'établissement de textes fiables, à la biographie des compositeurs, au jeu des influences, à la réception des œuvres. La musicologie théorique, au contraire, met l'accent sur l'aspect spéculatif et sur l'analyse détaillée des œuvres en fonction de techniques précises. Il semble donc à propos de réunir pendant une journée des gens œuvrant dans le domaine de la musicologie pour leur permettre de débattre des mérites relatifs des deux approches et de trouver un terrain d'entente qui permettrait d'en arriver à un type de musicologie «idéale ».

On trouvera plus bas les textes complets des prises de positions des quatre invités : (1) Jean-Michel Boulay, professeur de matières théoriques à l'Université d'Ottawa et auteur d'une thèse de doctorat sur la structure tonale dans Salome de Strauss ; (2) Paul Cadrin, professeur de matières théoriques à l'Université Laval et spécialiste de la musique de Szymanowski ; (3) Marc Honegger, musicologue et professeur honoraire à l'Université des sciences humaines de Strasbourg et responsable de la publication du Dictionnaire de la musique et du Dictionnaire des auvres de l'art vocal (Bordas) ; et (4) Jean-Jacques Nattiez, professeur de musicologie à l'Université de Montréal et auteur d'ouvrages sur la sémiologie, Proust et Wagner. Ces textes sont suivis d'un compte rendu du colloque, préparé par Vivianne Émond, étudiante au doctorat en musicologie à l'Université Laval.

\section{Prises de position des invités}

\section{Jean-Michel Boulay}

Le développement, fondamentalement américain, d'une "théorie de la musique », discipline que Jean-Jacques Nattiez appelle music theory en raison de son origine anglo-saxonne, a créé, comme vous le savez sûrement, maints remous au sein de la communauté universitaire nord-américaine. Les tensions auxquelles je fais allusion ont bien sûr d'abord été senties chez nos voisins du sud. Au Québec, ce n'est que depuis peu que les musicologues se sont intéressés de façon sérieuse à cette façon d'aborder les problèmes du langage musical.

En effet, la «théorie », encore une fois dans sa définition nord-américaine, n'est représentée dans nos établissements d'enseignement supérieur franco- 
phones qu'à l'Université Laval (par Paul Cadrin). Je travaille moi-même dans un établissement qui, bien que frontalier et bilingue, n'en demeure pas moins ontarien, donc très proche du monde universitaire anglophone. C'est ainsi qu'au Département de musique de l'Université d'Ottawa, qui est légèrement plus petit, je crois, que l'École de musique de l'Université Laval, la musicologie théorique est aussi importante que la musicologie historique. Notre département compte, dans son corps professoral à temps plein, quatre jeunes spécialistes de la « théorie » et quatre musicologues historiens.

Ces dernières années, ma discipline, que j'appellerai maintenant « analyse musicale ", car ce terme me semble mieux convenir que l'usage français traditionnel, a été, à maintes reprises, mise au banc des accusés. Les attaques les plus visibles et les plus publicisées sont venues d'un groupe d'historiens fortement influencés par les méthodes de la critique littéraire, parmi lesquels on retrouve de grands personnages, comme Joseph Kerman, Leo Treitler et Lawrence Kramer' ${ }^{1}$. On reproche d'abord à l'analyse d'avoir envahi les écoles de musique et d'avoir attiré plusieurs des meilleurs étudiants de doctorat. On l'accuse également de se faire omniprésente et trop exigeante quant à la qualité du travail des étudiants. puisqu'il constitue une lutte de pouvoir au sein des départements, des écoles et des facultés universitaires. On ne saurait sousestimer cet aspect des choses : l'apparition d'une nouvelle discipline, alors que l'expansion du corps professoral est très limitée, amène automatiquement une remise en question des anciennes attitudes et donc une insécurité pour les personnes en place.

On reproche aussi à l'analyse musicale, et c'est là un reproche beaucoup plus fondamental, d'être une entreprise purement formaliste, fille d'une idéologie esthétique dépassée, et qui doit être remplacée par une critique herméneutique informée par l'histoire. De plus, selon les représentants de deux courants particulièrement populaires pour l'instant (du moins dans le monde universitaire anglophone), soit la musicologie féministe et la théorie culturelle marxiste, la musicologie théorique ne tiendrait pas assez compte des facteurs sociologiques et politiques et de leur influence à la fois sur le contenu des œuvres musicales et sur notre perception de celles-ci.

Cette vision de l'analyse comme démarche strictement formaliste et

1 Joseph Kerman, « How We Got into Analysis, and How to Get Out », Critical Inquiry 7 (hiver 1980) : 311-31 ; Joseph Kerman, Contemplating Music: Challenges to Musicology (Cambridge, Mass. : Harvard University Press, 1985); Leo Treitler, Music and the Historical Imagination (Cambridge, Mass. : Harvard University Press, 1989); Lawrence Kramer, Music as Cultural Practice, 1800-1900 (Berkeley: University of California Press, 1990); Lawrence Kramer, " Haydn's Chaos, Schenker's Order; or Hermeneutics and Musical Analysis : Can They Mix ?", 19th-Century Music 16, n 1 (été 1992) : 3-17; Michael Cherlin, "Why We Got into Analysis and What to Get Out of It ", Theory and Practice 11 (1986) : 53-74. 
taxinomique est, selon moi, le résultat d'un malentendu. Bien sûr, je ne dis pas qu'on ne peut trouver de nombreux exemples frappants de bête réductionnisme dans la littérature théorique récente, surtout quand elle porte sur ce que mon maître, William Benjamin, appelle les deux "grandes orthodoxies » de la discipline, la théorie schenkérienne et la théorie des ensembles (set theory) ${ }^{2}$. Mais toute discipline, et donc, bien entendu, aussi la musicologie historique, a sa part de travail mal pensé et mal fait.

Plusieurs des malentendus entre historiens et théoriciens viennent d'une mauvaise compréhension, d'un côté comme de l'autre d'ailleurs, de ce qu'est l'analyse musicale. Comme le mentionnait Scott Burnham dans un numéro récent de 19th-Century Music, on a trop souvent fait l'erreur de présenter la « théorie » comme l'équivalent musical de ce qu'est la grammaire structurelle pour la langue, donc comme une discipline qui ferait fi des considérations sémantiques $^{3}$. Or, une analyse qui veut déboucher sur une théorie d'une œuvre musicale, c'est-à-dire une analyse qui nous dit autre chose que « cette musique est évidemment sensée, bien que nous ne dirons rien de son sens », ne saurait, bien sûr, oublier la question de la signification.

J'aimerais éclairer mon propos par un exemple. On a beaucoup attaqué les théories schenkériennes parce qu'elles donneraient trop d'importance aux niveaux plus profonds de la structure musicale, niveaux qui sont, selon Schenker, régis par des modèles abstraits de la conduite des voix. On a accusé Schenker de ne pas accorder assez d'importance à la surface de la musique, à ses ambiguiités, à ses points de ruptures, de tomber dans un mécanisme refusant « l'interprétation ». C'est bien mal lire ses analyses. Lorsqu'on prend la peine de s'y attarder sérieusement et de lire de près le texte explicatif dans sa version originale (au lieu de simplement se contenter de regarder les figures), on voit à l'œuvre un cerveau fascinant, réfléchissant sur les significations profondes, aussi bien sémantiques que structurelles, des événements musicaux ${ }^{4}$. Dans ses analyses, Schenker ne procède pas toujours dans la même direction, c'est-àdire soit par génération, soit par réduction, comme voudraient nous le faire croire plusieurs des soi-disant « schenkériens » américains. Schenker se promène entre les différents niveaux, sans adhérer de façon inflexible à une méthodologie inébranlable, tentant toujours de découvrir les chemins génératifs nous menant

2 William E. Benjamin, « Schenker's Theory and the Future of Music », Journal of Music Theory 25, $\mathrm{n}^{\circ} 1$ (printemps 1981): 171.

3 Scott Burnham, «The Criticism of Analysis and the Analysis of Criticism », 19th-Century Music 16, ñ 1 (été 1992) : 70-76.

4 Pour un bon exemple de ce type d'analyse, voir Heinrich Schenker, « Johann Sebastian Bach : Sechs Sonaten für Violine, Partita III (E-Dur), Präludio », dans Das Meisterwerk in der Musik : ein Jahrbuch 1 (1925) : 75-98 (réimpression; Hildesheim et New York : Georg Olms, 1974). 
d'un niveau à un autre, s'étonnant parfois d'une trouvaille harmonique, ou s'émerveillant souvent devant l'originalité d'un passage. Ce qui, pour lui, reste toujours inchangé, c'est le modèle le plus profond de la structure.

En fait, le principal problème des théories de Schenker, c'est qu'elles ont été disséminées par des interprètes souvent beaucoup plus doctrinaires que lui et, la plupart du temps, beaucoup moins bien préparés musicalement. En effet, il n'y a rien de plus rassurant pour un musicien médiocre que de se cramponner à un modèle inflexible et d'éviter ainsi l'incertitude du processus créateur et les ruptures qui sont à la base même de toute œuvre musicale.

Un phénomène identique se produit dans l'analyse des œuvres post-tonales. Ici, les dangers de succomber à la peur du vide sont encore plus grands. La contextualité du langage, l'absence d'une syntaxe partagée et la présence encombrante de multiples textes pseudo-analytiques, entrevues et esquisses des compositeurs eux-mêmes sont autant d'éléments qui peuvent faire déraper le processus analytique vers une sorte de « composition à l'envers ». On oublie alors le but premier de l'entreprise, qui est de réfléchir sur l'œuvre musicale et sur le fait qu'elle change nos vies.

C'est là la dernière idée que je voudrais développer. L'analyse est le seul moyen dont les musiciens disposent aujourd'hui pour tenter de comprendre le pourquoi de ce phénomène apparemment mystérieux que représente l'action profonde de la musique sur notre compréhension du monde et de nous-mêmes. Donc, pour arriver un jour à une analyse vraiment convaincante de la musique, on devra mieux comprendre le lieu musical de la signification et son mécanisme. Pour ma part, je crois de plus en plus que c'est ici que la musique diffère beaucoup de la langue. En musique, une part importante de la signification repose dans la syntaxe, c'est-à-dire que, bien avant qu'entrent en jeu toutes sortes de considérations extra-musicales, notre réaction à la musique repose sur nos attentes, sur nos modèles intérieurs du comportement syntaxique.

Pour arriver à cette forme d'analyse sérieuse de la musique, musicologues théoriciens et musicologues historiens devront arrêter d'utiliser les généralisations pédagogiques qui sont à la base de leur formation. Le problème fondamental de l'étude sérieuse de la musique est, vu sous cet angle, partagé par tous les musicologues. Nous avons tous été formés plus ou moins de la même façon. Pour des raisons pédagogiques évidentes, nous avons appris à classer les événements musicaux (et ici je parle aussi bien d'événements harmoniques que d'événements formels) en catégories. C'est pourquoi, trop souvent (et peut-être qu'ici les musicologues historiens sont encore plus coupables que les théoriciens), nous discutons des œuvres musicales en les comparant à des modèles du niveau des traités élémentaires, issus de généralisations simples (je dirais même simplistes) à caractère purement pédagogique. 
Malheureusement, l'analyse musicale bien faite et la réflexion théorique sont des activités qui demandent beaucoup de temps et de persévérance. Les adeptes d'une « critique musicale » englobante doivent faire porter une grande partie de leurs efforts sur autre chose que la partition, ce qui semble les amener souvent à oublier qu'un regard trop rapide porté sur une œuvre peut parfois faire dérailler complètement l'interprétation, toute séduisante qu'elle soit. Je ne citerai qu'un exemple. Dans son livre Feminine Endings, Susan McClary, dans un chapitre sur la représentation musicale des femmes folles, écrit, au sujet des dernières mesures de Salome de Richard Strauss :

Hérode réagit à la triomphale conclusion, en do dièse mineur, du "Liebestod " de Salomé en ordonnant à ses gardes d'écraser la princesse sous leurs boucliers en do mineur. [...] Les transgressions sexuelles et chromatiques de Salomé sont si monstrueuses qu'elles semblent justifier, ou même exiger, l'emploi d'une violence extrême, afin que soit rétabli l'ordre social et tonal's.

Tout ceci est très attrayant, mais n'a pas grand chose à voir avec la structure tonale de l'opéra. Cette conclusion en $d o$ mineur, bien loin de restaurer un ordre tonal, vient plutôt le perturber une dernière fois. Ici, do mineur confirme l'échange de rôles entre Salomé et Hérode et la réunion ultime de Salomé et de Jochanaan dans la mort. Du même coup, cette conclusion vient, une dernière fois, compléter un processus d'encerclement chromatique de la tonalité principale, processus qui est lui-même à la base de la structure tonale de cette carve ${ }^{6}$. Mais pour bien comprendre la place de la tonalité de do dans cette œuvre de Strauss, il faut l'avoir étudiée de près, je dirais même de très près, et cela, encore une fois, prends du temps (j'y ai passé moi-même quelques années...).

Ma réponse à la question du jour sera donc la suivante : la coexistence est certes possible, mais nous ne devrions pas nous contenter de coexister. Nous n'arriverons à mieux comprendre le mystère de la musique que quand nous pourrons communiquer entre nous sans avoir recours à des généralisations simplistes et à des catégories du niveau le plus élémentaire. Tout ceci ne se fera qu'au prix d'un travail immense et d'une intense réflexion, accompagnés d'une étude très minutieuse et ouverte du répertoire. Mais, vous en conviendrez certainement, le prix en vaut la chandelle.

5 Susan McClary, Feminine Endings : Music, Gender, and Sexuality (Minneapolis : University of Minnesota Press, 1991), 100.

6 Sur la structure tonale de Salome, voir Jean-Michel Boulay, "Monotonality and Chromatic Dualism in Richard Strauss's Salome » (thèse de doctorat, University of British Columbia, 1992), 12-54. 


\section{Paul Cadrin}

Musicologie historique et musicologie théorique : une coexistence est-elle possible ? Le titre du colloque qui nous réunit fait évidemment allusion aux difficultés d'émergence que la musicologie théorique a connues au cours des dernières années. Un historien des sciences faisait remarquer que, quand un nouveau paradigme de pensée fait son apparition, ce n'est pas nécessairement par la vigueur de ses démonstrations qu'il s'impose, mais souvent par l'épuisement des ténors de l'ancien système. Si cette hypothèse devait se vérifier dans notre cas, il faudrait d'ores et déjà prédire la victoire de la musicologie théorique et simplement recommander à ses tenants de faire preuve d'un peu de patience.

Ce serait un grand malheur pour tous, théoriciens comme historiens, car aucun des deux domaines ne saurait prospérer sans l'autre. Un système théorique qui couperait ses liens avec les données de l'histoire dégénérerait à court terme dans la sophistique. Une histoire qui voudrait se constituer en marge des données théoriques aurait inévitablement tendance à se confiner à l'étude de phénomènes paramusicaux. Cen'est donc pas d'une simple coexistence qu'il faut parler, mais d'une complémentarité essentielle.

Si telle est la situation dans la sérénité des principes, comment expliquer les tensions actuelles ? Principalement, je crois, parce que nous n'avons pas affaire à la musicologie historique ou à la musicologie théorique, mais à des musicologues qui s'identifient, soit comme historiens, soit comme théoriciens, donc à des personnes avec toutes leurs richesses et leurs limites. Dans la situation actuelle, les premiers se sentent peut-être menacés dans l'exercice d'un pouvoir traditionnel, alors que les autres se voient imbus d'une légitimité scientifique que leur confère la vigueur intellectuelle de leur domaine. Il importe ici de bien identifier les protagonistes. Je vais donc m'attacher à tracer le portrait'du musicologue théoricien, tout en laissant à mes collègues historiens le soin de tracer leur propre portrait.

Dans le monde francophone, le théoricien est très souvent identifié avec celui qui pratique l'analyse. Dans toutes les provinces du monde de la musique, on pratique depuis toujours l'analyse. Qu'on soit historien, critique, compositeur, interprète ou pédagogue, on est sans cesse appelé à exercer une certaine forme d'analyse des œuvres pour réaliser ses objectifs. Cependant, lorsque cette activité fait l'objet d'une spécialisation, elle prend une coloration particulière qui la distingue de l'analyse pratiquée par les autres professions de la musique. Si tout théoricien se doit de pratiquer l'analyse, toute personne qui fait de l'analyse ne devient pas théoricienne pour autant. À quoi reconnaît-on le véritable théoricien, la véritable théoricienne? 
Je relève cinq critères principaux : trois positifs et deux négatifs. Les critères positifs pourraient s'appeler l'analyse comme passion, l'analyse comme interprétation, l'analyse comme remise en question. Les critères négatifs, pour leur part, s'intituleraient le refus de faire la chasse aux sorcières, d'une part, et, d'autre part, l'horreur des « trucs».

\section{L'analyse comme passion}

Le théoricien pratique l'analyse d'abord et avant tout par passion pour l'analyse, parce que celle-ci lui révèle toutes sortes de richesses qui risquent de rester cachées à celui qui se contente d'écouter ou d'interpréter l'œuvre. Comme toute personne passionnée, le théoricien s'attache à ce qu'il perçoit comme étant ce qu'il y a de meilleur. Concrètement, cela signifie qu'il ne saura pas faire une analyse valable d'une œuvre qu'il considère comme médiocre et qui ne l'intéresse pas vraiment. Par contre, la flamme nourrissant la flamme, plus il s'attachera à une œuvre, plus il saura en discerner les trésors.

C'est parce qu'il pratique l'analyse par passion que le théoricien privilégie l'approche immanente, un sujet que le professeur Nattiez pourrait développer beaucoup mieux que moi. Qu'il me suffise ici de dire qu'avant d'être comparée avec ses contemporaines, ou avec celles qui l'ont précédée ou suivie, l'œuvre est confrontée à elle-même. C'est l'œuvre elle-même qui doit suggérer au chercheur les outils appropriés à son analyse. La passion de l'analyse passe donc d'abord par le fait de mettre l'œuvre dans son individualité au centre de l'activité analytique.

Cependant, l'analyste ne manquera pas de se munir des balises que peut lui fournir un système théorique. Le rôle de ce système ici est uniquement de lui permettre de savoir poser les bonnes questions à l'œuvre. Ce système sera donc d'autant plus efficace qu'il transcendera les traits stylistiques d'un genre ou d'une époque. Le choix de ce système ne sera pas fondé exclusivement sur des considérations tirées de l'histoire de la musique. Au contraire, le théoricien n'hésitera pas à faire appel aux ressources de la linguistique, des mathématiques, de la psychologie de la perception, de l'anthropologie, etc., s'il pressent que ces disciplines peuvent lui fournir des pistes valables pour la tâche qu'il entreprend.

C'est aussi parce qu'il pratique l'analyse par passion que le théoricien se place, par rapport à l'œuvre, dans un position qui le rapproche davantage du compositeur que de l'historien. Au moment où le compositeur écrit l'œuvre, l'œuvre existe nécessairement pour lui dans le présent. L'histoire en tant que telle n'est pas outillée pour rendre compte du présent ; elle ne cherche pas à comprendre les œuvres en tant qu'appartenant à l'aujourd'hui. Le présent ne peut être vraiment compris que d'un point de vue synchronique. Il n'est donc pas étonnant que, au cours des développements récents de la musicologie 
théorique en Amérique du Nord, les figures de proue du mouvement aient été issues du milieu des compositeurs, et non de celui des historiens.

\section{L'analyse comme interprétation}

Le théoricien a compris que l'analyse est une forme d'interprétation, au même titre que l'interprétation instrumentale. Ceci suscite deux types de réflexions, l'une sur l'autonomie de l'approche théorique et l'autre, sur le caractère provisoire de toute analyse.

Premièrement, l'analyse est une activité intellectuelle autonome, qui n'est pas au service de quelqu'autre discipline que ce soit, mais bien au service de l'œuvre musicale qu'elle cherche à mettre en valeur. Si l'analyse est bien faite, il est normal que l'interprète, le compositeur, l'historien, le psychologue et le pédagogue de la musique y trouvent leur profit, mais ce n'est pas là son but premier. Une ouvre vraiment significative peut être vue sous de nombreux angles, peut être lue de plusieurs façons ; l'analyse en est une. Ceci, soit dit en passant, répond à ceux qui craignent que l'analyse ne démythifie les grandes œuvres pour les réduire à des formules sèches. Si tel était le cas, il faudrait conclure, ou bien que l'œuvre n'était pas si riche qu'on le croyait spontanément, ou bien que l'analyse est ratée, ce qui est plus probable. En effet, une œuvre véritablement significative ne saurait sortir que grandie d'une analyse qui va jusqu'au bout de ses responsabilités, comme elle ressort grandie d'une bonne interprétation.

Deuxièmement, comme l'interprétation à l'instrument, l'analyse se fait dans un climat hautement subjectif et provisoire. Le véritable analyste est toujours prêt à poser d'autres questions à l'œuvre, à recommencer son travail selon un nouveau point de vue, à partir de nouveaux paramètres. Il n'y a pas plus d'analyse définitive qu'il n'y a d'interprétation définitive. À cet égard, on pourrait dire que, autant le travail du musicologue historien s'identifie avec la recherche des certitudes les mieux étayées, autant celui du théoricien est placé sous le signe du risque permanent.

\section{L'analyse comme remise en question des systèmes}

Le véritable théoricien est persuadé que tout ce qui est cohérent s'explique, et que plus l'explication elle-même sera formalisée, mieux la cohérence sera démontrée. C'est d'ailleurs la raison principale pour laquelle de nombreux analystes contemporains n'hésitent pas à faire appel aux ressources des mathématiques, qui peuvent leur fournir des modèles de formalisation absolument admirables.

Cependant l'analyste reste également conscient du fait que les systèmes 
théoriques, même les plus puissants, ont des limites ; aucun système ne saurait épuiser la réalité. Lorsque le théoricien se trouve confronté à une situation qu'il perçoit comme cohérente, mais qui semble contredire la théorie, il n'hésite pas à remettre la théorie en question. Parce que le système n'est pas une fin en soi, le théoricien le soumet constamment aux vérifications les plus rigoureuses pour en déterminer la validité dans chaque cas particulier. Du côté de l'œuvre, ce principe a pour corollaire qu'il n'existe pas de « licence du compositeur»; si elle existait, cette fameuse " licence », elle serait tout au plus le symptôme de la paresse de l'analyste.

L'analyse comme passion, l'analyse comme interprétation et l'analyse comme remise en question des systèmes, tels sont les trois traits de caractère positifs du véritable théoricien. Tournons-nous maintenant vers ses aspects négatifs. Je n'en relève que deux.

\section{Refus de faire la chasse aux sorcières}

Pour le théoricien, l'analyse n'est pas la « recherche des intentions du compositeur », ni même "l'art de retracer les processus de composition »; c'est là ce que j'appelle la chasse aux sorcières. L'analyste ne cherche pas à attribuer au compositeur d'autres intentions que celles qu'il a couchées sur le papier de la partition telle qu'elle nous parvient (en autant qu'on en a garanti l'authenticité auparavant, cela va de soi !). Franchir cette frontière en cherchant à retrouver des intentions plus ou moins cachées ne relève pas de la compétence du théoricien, mais plutôt de celle de l'herméneuticien. Ce dernier doit s'appuyer sur l'analyse, bien sûr, mais il pratique une activité intellectuelle qui est formellement distincte de celle-ci. Curieusement, comme nous venons de le voir, ce point de vue n'a pas pour effet d'éloigner le théoricien du compositeur, tout au contraire!

Il en va de même pour la recherche des processus de composition : il arrive trop souvent que le compositeur s'illusionne lui-même sur le rôle et l'efficacité des processus auxquels il fait appel. L'histoire, surtout celle de notre siècle, est remplie de cas de ce que j'appellerais des « dissonances » entre les intentions du compositeur, d'une part, et, d'autre part, le résultat sonore qui, seul, peut être considéré comme œuvre musicale. Ainsi, dès les années 60 , Xenakis choisit ses hauteurs à l'aide d'opérations mathématiques tellement complexes qu'il doit les confier à des ordinateurs ; cependant, lorsque les hauteurs ainsi calculées sont ensuite systématiquement affublées d'indications de glissando, il faut bien convenir que le résultat sonore n'a rien à voir avec les calculs, si savants soientils, et que le compositeur aurait pu atteindre le même résultat avec des moyens beaucoup plus simples. L'analyse de ces œuvres doit alors transcender l'examen des systèmes mathématiques qui sont censés les avoir engendrées. 


\section{Horreur des « méthodes » et des « trucs »}

Ces panacées cachent mal l'indigence intellectuelle de ceux qui les fréquentent. La véritable analyse exige l'implication personnelle complète de celui qui la pratique. Un des critères d'une analyse réussie, c'est que, à l'issue de sa démarche, l'analyste a mémorisé l'œuvre dans ses moindres détails, comme l'aurait fait un interprète, parce qu'il l'a reconstruite intérieurement. D'une certaine façon, on peut y voir l'application du principe de la table rase : idéalement, l'analyste doit aborder chaque œuvre comme si c'était la première fois de sa vie qu'il faisait une analyse. Il doit alors se cramponner de toutes ses forces à ses perceptions, à ses intuitions, et éviter à tout prix de se réfugier dans le confort des méthodes éprouvées et des trucs garantis par le fabriquant.

À partir de ces considérations, quelle réponse peut-on apporter à la question qui est au cœur du débat qui nous réunit ? Tout d'abord la coexistence de la musicologie historique avec la musicologie théorique est non seulement possible et souhaitable, elle est même nécessaire. Elle est nécessaire au musicologue théoricien, parce que, en vertu même du point de vue qu'il a choisi, sa perception de l'œuvre est intentionnellement appauvrie à certains égards. Il a besoin des lumières de l'historien pour ne pas perdre de vue le contexte dans lequel s'inscrivent les œuvres qu'il analyse. Par ailleurs, précisément parce qu'il adopte un point de vue synchronique, le théoricien peut rendre de précieux services à l'historien, qui a spontanément tendance à voir l'œuvre d'abord comme un maillon d'une chaîne, que ce soit celle de la production d'un compositeur particulier, ou bien celle d'un style ou d'un genre donné. L'historien risque alors de l'aborder avec des idées préconçues qui l'appauvrissent. Évidemment, le théoricien peut également rendre de précieux services aux interprètes, aux compositeurs et aux critiques, mais c'est là le sujet d'un autre colloque !

\section{Marc Honegger}

Tel qu'il est formulé-Musicologie historique et musicologie théorique : une coexistence est-elle possible ?-le thème de ce colloque fait apparaître l'existence supposée ou réelle d'une opposition tranchée entre deux aspects majeurs de notre discipline. Toutes les définitions de la musicologie concordent en ce qu'elles soulignent à la fois son unité et sa diversité. Dans sonDictionnaire pratique et historique de la musique (Paris, 1926), Michel Brenet s'exprime en ces termes : "Science de la musique, qui embrasse sa théorie et son histoire [...] Ses ramifications la rattachent aux ordres de connaissance en apparence les plus éloignés. Elle relève directement de la physique par l'acoustique, de la physiologie par l'étude de l'appareil vocal et de l'appareil auditif, de la 
psychologie par l'esthétique ; elle apporte, par l'étude des gammes et du folklore, une contribution notable à l'ethnographie ; dans la recherche des rythmes, elle rencontre la métrique et la cinématique ; du côté de l'histoire, elle est inséparable des études liturgiques, littéraires, nationales ; elle montre l'union de tous les arts et le rôle de la musique dans l'histoire des civilisations. Cette variété d'aspects attire de nos jours vers la musicologie un nombre sans cesse grandissant de savants, d'érudits, de lettrés et d'artistes, et provoque la spécialisation féconde de leurs travaux. »

Une réponse à la question qui nous est posée aujourd'hui se trouve donc implicitement contenue dans cette définition. Des ouvrages plus volumineux et plus récents n'ont fait que la développer sans en modifier le caractère : l'article «Musikwissenschaft " de l'encyclopédie allemande Die Musik in Geschichte und Gegenwart, rédigé par Walter Wiora et Hans Albrecht, et l'article "Musicology» de l'encyclopédie britannique The New Grove Dictionary of Music and Musicians, œuvre de Vincent Duckles aidé de plusieurs collaborateurs de renom. Bien que renchérissant sur l'énumération des composantes de la musicologie, aucune de ces deux études ne s'avise d'opposer l'aspect historique et l'aspect théorique, considérés comme complémentaires quoique nettement séparés dans chaque exposé. On peut donc se demander à la suite de quelle évolution on en est arrivé en quelques décennies à concevoir entre eux un rapport quasi conflictuel, même s'il entre de la provocation dans la formulation du thème de ce colloque.

Il y a peu de temps encore, l'aspect historique et l'aspect théorique pouvaient être assumés par la même personne. De ce fait l'adéquation de l'un à l'autre était assurée, tandis qu'un bilan synthétique s'avérait à tout instant possible. Depuis quelques décennies, on en est venu—surtout dans le Nouveau Monde, me semble-t-il-à accorder priorité à l'étude de l'œuvre musicale en ellemême, indépendamment de toute considération d'ordre historique ou culturel, et à développer une analyse de type structuraliste, issue par analogie de la linguistique, élaborant du même coup des méthodes nouvelles qui font appel entre autres à la statistique et aux mathématiques. Devenue une discipline en soi, elle mène désormais une vie autonome, détachée des perspectives traditionnelles de la musicologie. On ne saurait nier qu'elle a ouvert des perspectives nouvelles à la connaissance du fait musical dans sa matérialité. Quoi qu'il en soit du destin de cette discipline de nature théorique, pas plus aujourd'hui qu'hier la musicologie traditionnelle-celle qui s'attache à l'évolution de la musique occidentale - ne peut se passer de l'analyse, ni celleci d'un cadre historique. La collaboration de ces deux disciplines s'impose donc, mais à condition que soient respectées un certain nombre d'exigences fondamentales, étant bien entendu qu'il existe d'autres points de vue que celui de l'historien et du musicien formé aux disciplines occidentales. 
En premier lieu, il faut accepter l'idée que la musique est susceptible de véhiculer d'importantes données extra-musicales. Dans ce cas extrêmement fréquent, elle n'est que la forme sonore donnée à des idées ou à des sentiments qui viennent d'ailleurs. Or, l'idée conditionnant la forme, une analyse qui ne tiendrait pas compte du sens ou de la fonction de l'œuvre risquerait d'aboutir à un jeu gratuit, utile dans le meilleur des cas à la pédagogie musicale, mais d'une pertinence limitée par rapport à l'œuvre elle-même. Il semblerait d'ailleurs que la plupart des analyses récentes s'attachent à des œuvres instrumentales, moins chargées en connotations, plutôt qu'à des œuvres vocales.

L'analyse ne peut guère être un but en soi. Si cela devait être le cas, elle deviendrait à la limite une création de l'esprit, mais privée de toutes les séductions et de tous les pouvoirs de la musique. Il apparaît donc indispensable qu'elle fasse soigneusement la part de l'essentiel et de l'accessoire afin de dégager des éléments concourant à une synthèse. Ceci devrait empêcher de la limiter à une simple nomenclature d'éléments constitutifs.

S'attachant à une œuvre du passé, toute analyse devrait tenir le plus grand compte des données d'époque, tout particulièrement en ce qui concerne la théorie musicale, sinon le risque serait grand d'aborder l'étude d'une œuvre ou d'un style avec des critères inadaptés. On est loin du temps où la musique classique paraissait la référence obligatoire de toute autre musique et où les maîtres antérieurs au XVIII ${ }^{e}$ siècle étaient considérés comme des primitifs par rapport à Bach ou à Mozart, qui représentaient l'accomplissement de l'évolution musicale en Occident. Et cependant ce grave défaut de méthode réapparaît lorsque l'on prétend analyser une œuvre du début de la Renaissance en numérotant les intervalles en fonction du total chromatique sans tenir compte $\mathrm{du}$ fait que, jusque vers le milieu du $\mathrm{XVI}^{\mathrm{e}}$ siècle, la totalité de la musique s'inscrit dans un système heptatonique et que l'usage des notes chromatiques, des " feintes », est de caractère essentiellement ornemental à l'exception du bémol susceptible d'affecter la note si. En outre, se priver des données historiques et culturelles serait se placer volontairement dans la situation de l'archéologue de la préhistoire qui, étudiant des vestiges de civilisations disparues et considérant des objets non strictement utilitaires ou des représentations de nature symbolique ou religieuse, ne peut apprécier que leur forme sans être à même d'en déchiffrer le sens. Dans le cas de la musicologie, une telle attitude serait une régression.

Il faut aussi se garder d'un intellectualisme qui pousse à privilégier des œuvres à haut niveau d'abstraction ou comportant une forte proportion d'éléments codés, alors que d'autres œuvres non moins importantes nécessitent une approche plus intuitive ou plus sensible. Il est évident que les Variations pour piano de Webern offrent à l'analyste une matière plus gratifiante que Jeux de Debussy par exemple. 
Il faut en outre se méfier de la notion de progrès en art et ne faire d'aucun système théorique le garant de l'intérêt ou de l'actualité d'une œuvre. On ne peut se défaire de l'impression que le développement de l'analyse structurale se trouve étroitement associé à celui du dodécaphonisme et du sérialisme. La démonstration de la logique interne d'une œuvre y vient à propos occulter l'arbitraire d'un choix théorique, celui du système des 12 sons.

Enfin, quels que soient la nature et les buts de toute analyse du phénomène musical, il n'y a aucun intérêt à enfermer la discipline dans un cercle restreint d'initiés. Il faut pour cela éviter l'emploi d'un langage hermétique, emprunté à la philosophie ou à la linguistique, mais avoir constamment le souci d'une expression claire, qui ne tombe ni dans la virtuosité ni dans la technicité, afin de rester accessible à tout musicien curieux des choses de son art.

Ceci dit, on comprendra que ma réponse positive à la question posée soit teintée de fortes réserves. Je souhaite vivement que l'avenir permette de les atténuer si ce n'est de les supprimer, mais je crains qu'il faille désormais nous faire au morcellement de la musicologie, ainsi qu'à un pluralisme de points de vue. De toute façon, il n'est pas dénué d'intérêt que l'analyse attire notre attention sur ce qui devrait rester essentiel, l'œuvre musicale, mais revêtue de son sens le plus complet.

\section{Jean-Jacques Nattiez}

Je suggère qu'on se mette tout d'abord d'accord sur les termes. En principe, on n'utilise pas en français l'expression « musicologie théorique »; il s'agit de la traduction de l'anglais music theory. Le New Grove est très clair sur ce que l'expression recouvre. L'article « Theory » commence ainsi : "Theory is now understood as principally the study of the structure of music. » D'un côté donc, la musicologie historique qui, comme nous le rappelle Marc-André Roberge, comprend entre autres choses l'étude de la vie musicale, la description des sources, l'établissement des textes, les biographies, les influences et la réception des œuvres. De l'autre, une approche qui présente trois caractères : la music theory est à la fois analytique, immanente (ou structurale) et synchronique.

Je dirai d'emblée que je me situe dans une perspective œcuménique : pour moi, contrairement à l'usage de nos collègues anglophones pour lesquels « musicology » est pratiquement synonyme de « musicologie historique », il y a la musicologie, étude scientifique du fait musical, avec ses différentes branches (ou champs d'activité) commel'histoire, l'analyse, l'ethnomusicologie, la psychologie et la sociologie de la musique, et les diverses méthodes de ces branches. J'insiste sur ce dernier point car, comme nous allons le voir, chaque paradigme méthodologique constitue le fait musical en un objet différent. 
Dans cette perspective, on comprend que je n'aie aucune difficulté à chercher avec vous où il peut $y$ avoir coexistence et terrain d'entente entre musicologie historique et music theory. Mais pour ce faire, il convient de rappeler, pour commencer, les raisons de la distinction, voire du divorce.

Elles me semblent de deux ordres : épistémologiques et institutionnelles.

Rappelons-nous le texte fondateur de la musicologie, l'article Umfang, Methode und Ziel der Musikwissenschaft publié par Guido Adler en 1885 dans le premier numéro du Vierteljahrschrift für Musikwissenschaft. Adler distingue entre la musicologie historique et la musicologie systématique, c'est-à-dire la recherche des lois fondamentales, en deçà de la diachronie, dans l'harmonie, le rythme et la mélodie. On le voit, ce n'est pas exactement la distinction qui nous occupe ici, puisque l'analyse des structures n'apparaît pas, chez Adler, comme séparée de l'approche historique, mais il pose la distinction entre le synchronique et le diachronique. Celle-ci a été, bien sûr, systématisée par Saussure qui affirme que chacune des deux approches définit deux ordres de réalité selon que l'on étudie les phénomènes du point de vue de leur évolution ou qu'on les envisage comme constitutifs d'un système. Schenker est un cas typique au début du siècle. Tout comme Schönberg, sa préoccupation théorique est celle d'un compositeur-théoricien qui constitue le système tonal en une immense couche synchronique de trois siècles. Je ne crois pas qu'il y ait eu un lien direct entre la pensée saussurienne et l'évolution de la musicologie, mais la distinction était dans l'air du temps. Le point important à retenir, c'est que les deux points de vue, le synchronique et le diachronique, constituent le fait musical en deux types d'objet distinct.

Le développement autonome de l'analyse musicale, avec son corpus de méthodes propres, comme en témoigne l'article «Analysis » de Bent dans le New Grove, aura été tel ces dernières décennies que, d'épistémologique, la distinction est devenue institutionnelle. Pour s'en tenir à l'Amérique du Nord, l'American Musicological Society, unique à l'origine, a d'abord été délaissée par les fondateurs de la Society for Ethnomusicology, puis par ceux de la Society for Music Theory. Je ne m'attarderai pas à ce clivage institutionnel, mais il est important de le mentionner, car il se retrouve dans les universités et dans les revues, et renforce ainsi la distinction fondée à l'origine sur une distinction épistémologique.

C'est donc elle qu'il convient d'interroger. L'opposition synchronie/ diachronie est-elle justifiée?

L'approche synchronique se justifie par la notion de système. Où peut-on le trouver? Dans des lois générales qu'il est parfaitement légitime de rechercher, mais qui font que, parfois, l'analyse d'une œuvre musicale a l'allure d'une tautologie : elle consiste à retrouver le système dans chaque œuvre. C'est un des 
reproches qui a été fait à Schenker. Mais l'approche synchronique implique aussi le systématique en un sens un peu différent : l'idée que l'œuvre doit faire l'objet d'un balayage systématique, d'une décomposition en ses différentes parties. Cela est vrai depuis l'organicisme des débuts de l'analyse musicale jusqu'aux différentes activités analytiques qui se réclament explicitement du structuralisme ou qui ont subi son influence.

L'approche synchronique est-elle justifiée ? Oui. Il est légitime de regarder si la musique tonale obéit à des lois, et de décrire-j'insiste sur le mot décrirede quoi est fait un style ou comment une œuvre est organisée. Admettons donc qu'il y a une autonomie du synchronique.

Mais en ce qui concerne les traits d'une œuvre pertinents par rapport à un style d'époque ou les traits spécifiques d'une pièce, en rester au niveau synchronique n'est qu'une des deux manières d'aborder le fait musical : en effet, la question de l'origine de ce style et de la spécificité d'une œuvre peut être immédiatement posée, et c'est celle qui vient spontanément à l'esprit de l'historien.

Pour l'instant, même lorsque les structures de l'œuvre sont abordées d'un point de vue diachronique, nous sommes encore à l'intérieur de l'analyse musicale : on peut faire une histoire de la transformation des langages, des formes et des styles musicaux sans faire intervenir ce qui est le propre de l'histoire de la musique : l'étude des sources, les reconstitutions philologiques, les biographies, etc.

Demandons-nous alors si, symétriquement, il y a une autonomie du diachronique. L'établissement du texte devrait précéder toute approche analytique, il est premier. On peut écrire une biographie répondant à toutes les normes et précautions de la science historique sans citer une seule note de musique. Il en va de même dans les ouvrages d'histoire. Pas un seul exemple musical dans les 427 pages de La musique romantique d'Alfred Einstein (1959), dans les 895 pages de l'histoire de la musique de Lucien Rebatet (1969), dans L'histoire de la musique en Europe de Brigitte François-Sappey (1992).

On le voit : du point de vue épistémologique, rien ne s'oppose à ce qu'un chercheur découpe son objet selon le clivage entre synchronie et diachronie. Les deux autonomies sont légitimes. Où ça ne va plus, c'est quand on prétend que la seule musicologie légitime soit l'historique ou l'analytique.

Les exemples de ces positions abondent. Adorno, repris par Dahlhaus, affirme : " Musik ist geschichtlich durch und durch. » En dehors de l'histoire, point de salut. À l'inverse, si l'on croit que tout est dans les structures, il est facile de céder à l'impérialisme de l'immanence. Si le débat entre le synchronique et le diachronique est si vif, c'est parce qu'il se double d'un autre dont l'enjeu est grave, parce que de nature ontologique, et qui soulève la question : qu'est- 
ce que la musique ? Un jeu de formes en mouvement, comme l'affirmait Hanslick, ou une forme symbolique qui renvoie au vécu du compositeur, qui éveille celui des auditeurs, et qui a partie liée à l'histoire, à la société et à la culture ? Dans le premier cas, on fait confiance à la forme, à la substance musicale, dans le second, aux documents qui lui sont extérieurs. Un exemple : Meyer rejette l'utilité de l'analyse des esquisses et ne croit à l'étude des processus compositionnels que d'un point de vue cognitif, faisant davantage confiance à l'établissement de lois qu'à l'étude de manuscrits souvent fragmentaires. À l'inverse, Kerman et le music criticism n'envisageraient pas de séparer l'étude des œuvres de leur environnement social, culturel et historique. Mais il y a une différence importante entre les deux tendances dans la façon d'aborder les œuvres : dans les travaux du music criticism, les œuvres ne sont pas décrites de façon systématique comme peut le faire un praticien de la music theory, quelles que soient les méthodes utilisées.

C'est qu'en effet, le clivage ontologique se double d'un clivage méthodologique : quel type de discours devons-nous utiliser pour rendre compte des faits musicaux ? Le discours historique pratique ce qu'une de mes étudiantes, Élisabeth Bertrand, appelait d'une expression québécoise bien efficace, le " pigisme ". Au contraire, pour l'immanentiste et le synchroniste, si je peux les appeler ainsi, l'être de l'œuvre musicale réside dans sa structure. Il faut donc décrire le détail de ses composantes musicales et de leurs interactions.

$\mathrm{Si}$, comme je le crois, la question qui nous est posée aujourd'hui porte, en fait, sur la possibilité d'établir un lien entre les informations externes (biographie, esquisses, histoire, écrits de musiciens, correspondance) et les structures immanentes des œuvres, je crois que la difficulté essentielle vient de ce que, la plupart du temps, les approches historiques-et plus généralement socioculturelles-sont holistes : elles sont, pour reprendre le mot de Michel Imberty, " suprasegmentales "; elles portent sur de grands ensembles sans chercher à justifier, à expliquer le détail des œuvres et de leur organisation hiérarchique, alors que les approches synchroniques les ont exploré systématiquement. Dès lors, y a-t-il nécessité de mettre en rapport les deux types de discours? Et si oui, cela est-il possible?

À la première question, je n'hésite pas à répondre positivement. Épistémologiquement, la musicologie traite de ce que Molino appelle, en paraphrasant Marcel Mauss, le fait musical total. L'œuvre a une réalité matérielle et immanente dont les structures sont analysables, mais qui résultent de processus poïétiques. Or, dans le poïétique, nous trouvons aussi bien les stratégies compositionnelles qui sont de l'ordre du technique, du psychologique, du cognitif, que des déterminations plus larges, historiques, sociales et culturelles. Or, le passage de l'immanent au poïétique, donc à l'historique, signifie passage 
du descriptif à l'explicatif. Si l'on remonte aux informations fournies par l'histoire-ou à la sociologie ou à l'anthropologie de la musique, le problème est le même-, c'est pour tenter d'expliquer le pourquoi des structures et des formes. La difficulté, c'est que le poïétique, précisément parce qu'il est de l'ordre du processus, n'est pas nécessairement lisible dans l'immanent.

Je crois donc que le point de contact entre approche historique et music theory est possible si l'on tente de répondre à la question : quelle pertinence poiétique puis-je attribuer aux structures? Méthodologiquement, je crois qu'on ne peut répondre à cette question qu'à la condition de séparer les paramètres constitutifs de l'œuvre-motifs, phrases, structures mélodiques, harmoniques, rythmiques, formelles, etc.-et ses diverses dimensions, sémantiques et stylistiques, et de tenter de mettre en rapport ces composantes avec les informations fournies par l'approche historique « externe » et cela, selon un mouvement perpétuel de va et vient : en allant inductivement des structures à l'histoire ou en projetant l'histoire sur les structures pour les réinterpréter.

Quand on tentera, d'une façon ou de l'autre, de mettre en rapport ces paramètres avec une détermination poïétique, en particulier d'ordre historique, certains aspects mis en évidence par la description immanente échapperont à cette mise en relation, et ce, selon trois cas de figures possibles :

(1) soit parce que les traits sont de nature universelle (on échappe alors à l'historique ; on est dans le biologique, le cognitif, l'anthropologique);

(2) soit parce que ce que l'on a identifié au niveau immanent n'est pertinent que du point de vue perceptif (les stratégies compositionnelles et les stratégies perceptives ne se correspondent pas nécessairement, nous le savons aujourd'hui);

(3) soit parce qu'on n'est pas capable de trouver un lien de causalité entre l'historique et l'immanent.

On retrouvera alors la part d'autonomie que l'immanent et le structural possèdent par rapport à l'historique.

Y a-t-il une musicologie «idéale », selon le mot de Marc-André Roberge, qui permettra à la musicologie historique et à la music theory de trouver un terrain d'entente ?Cela est non seulement légitime, puisque dans l'établissement de la relation entre la structure et l'histoire, il s'agit de passer du descriptif à l'explicatif, mais cela est possible, à condition de définir la passerelle relativement étroite où, de manière précise, pour tel et tel paramètre, on réussit à définir un lien explicite de causalité. 


\section{Compte rendu du colloque}

\section{Vivianne Émond}

Si le thème du colloque laissait présager un débat à tendance polémique, les positions fort nuancées des invités ont permis de cerner rapidement un point de convergence : non seulement la coexistence de la musicologie historique et de la musicologie théorique est-elle possible mais il faut aller plus loin en pensant en terme de complémentarité pour l'avenir. La tentation de reprendre certaines accusations et de tenter d'y répondre était grande du côté des théoriciens, tout comme pour les musicologues de tendance humaniste de rappeler les fondements de la division-sinon du divorce-, mais les invités ont surtout cherché à faire valoir les aspects positifs permettant d'entrevoir un rapprochement des branches de la musicologie. On a aussi bien sûr fait grand état des problèmes énormes de critères et de méthodes qui séparent les deux approches, le tout teinté de remarques sur la terminologie française (musicologie théorique, ou music theory, ou analyse musicale, ou musicologie systématique).

L'échange qui a suivi entre les invités et la trentaine de participants sur la question à débattre (et sur bien d'autres sujets qui auraient pu donner lieu à autant de rencontres) a mis en évidence de nombreuses situations que vivent les musicologues et les théoriciens aujourd'hui. Il y a eu notamment une réflexion intéressante sur la situation de la musicologie en Amérique du Nord par rapport à l'Europe et sur les différentes approches qui opposent les pays de tradition francophone et ceux de culture anglo-saxonne, avec entre autres le phénomène nord-américain de l'importance croissante de l'enseignement de la théorie dans les universités. Le clivage culturel qui sépare par exemple la musicologie française de la musicologie américaine est une réalité qui tend à s'atténuer pour certains, qui repose sur des clichés pour d'autres, ou encore qui se résume à une formation culturelle différente qui conditionne le goût. Il va sans dire que la présence de quatre invités issus de l'une ou l'autre des traditions, tant par leur formation que par leurs activités professionnelles, a apporté une dimension toute particulière au débat —et le tout sans l'habituelle barrière des langues. Tout comme les interventions sur la préparation différente des étudiants dans les universités européennes et nord-américaines, sur l'accessibilité des sources et même sur un certain manque de confiance dans sa propre culture ont jeté les bases d'un dialogue animé entre les étudiants et les professeurs.

La question capitale concernant le développement de l'analyse structurelle en fonction de la nécessité de tenir compte du contexte et des données historiques a aussi été posée de façon très claire. Sur cette lancée, le cœur du problème est rapidement apparu avec le renouvellement possible de la 
musicologie historique vers des voies nouvelles et l'évolution de la musicologie théorique qui ouvre de nouveaux horizons. Loin de vouloir tomber dans d'autres dogmatismes, on a préféré souligner la prise de conscience des théoriciens qui réagissent maintenant aux travaux réalisés, sans prétendre à la synthèse ou à la finalité d'une analyse mais en apportant une contribution. On a aussi rappelé que l'histoire évolue et que chaque époque propose de nouvelles lectures et pose de nouvelles questions selon l'esprit du temps. On peut reprendre des champs de recherche sous un angle différent, s'engager dans des voies inexplorées ou méconnues, comme l'étude des pratiques musicales, ou encore faire l'histoire de la musique récente. L'évolution des tendances, considérées en dehors des préjugés ou des accusations, laisse entrevoir des perspectives intéressantes de complémentarité. C'est sans doute dans cette direction que semble se trouver la véritable ouverture vers une compréhension mutuelle des mérites de chaque approche, dont le résultat pourrait être des biographies intégrant des éléments d'analyse beaucoup plus poussés et des analyses d'œuvres qui ne sont pas détachées de l'environnement propre à chaque époque ou qui excluent systématiquement toute donnée extra-musicale.

Des faits, des explications, des exemples concrets et de nombreuses questions en suspens sur un possible terrain d'entente entre la musicologie historique et la musicologie théorique ont donc permis d'établir un dialogue sinon fructueux du moins ouvert sur l'avenir. Le ton modéré des propos des invités et des participants et l'optimisme de plusieurs, avec ce réel désir de rapprochement, sinon de fiançailles entre la musicologie historique, qui a atteint sa maturité, et la musicologie théorique, qui est en voie de l'atteindre, laissent en tout cas présager d'autres réflexions en profondeur sur un sujet de la plus grande actualité. Quoi qu'il en soit, on peut aisément conclure de cette trop brève séance que ce fameux musicologue doué de l'esprit de synthèse souhaité par Marc Honegger ne pourra voir le jour qu'à condition de réussir à dominer des disciplines très différentes.

\section{Résumés}

\section{Jean-Michel Boulay}

D'après ses accusateurs, qui sont en général historiens de la musique, l'analyse musicale se serait rendue coupable de négligence : on aurait oublié de tenir compte des grandes lignes de développement historique, de l'expérience esthétique, du contexte sociologique, du cadre idéologique, et de bien d'autres choses encore. Plutôt que cette polémique stérile, il convient de se faire l'apôtre de la discipline en tentant d'expliquer, à l'aide d'exemples, ce que devrait être l'étude sérieuse des œuvres musicales comme réalisations d'un certain langage, 
et ce à partir de deux postulats de base : tout d'abord, l'analyse musicale ne saurait être une composition en rétrograde ; deuxièmement, elle devrait avoir pour but de rendre visible l'individualité de l'œuvre.

\section{Paul Cadrin}

Un système théorique qui couperait ses liens avec les données de l'histoire risquerait fort de dégénérer en sophistique et une histoire qui voudrait se constituer en marge des données théoriques se verrait rapidement confinée à l'étude de phénomènes paramusicaux. Ce n'est donc pas d'une simple coexistence qu'il faut parler, mais d'une complémentarité essentielle. Les tensions actuelles s'expliquent parce que nous n'avons pas affaire à la musicologie historique ou à la musicologie théorique, mais à des musicologues qui s'identifient, soit comme historiens, soit comme théoriciens, les uns se sentant menacés dans l'exercice d'un pouvoir traditionnel, les autres se sentant imbus d'une légitimité scientifique que leur confère la vigueur intellectuelle de leur domaine. La coexistence sera donc possible dans la mesure où les uns et les autres reconnaîtront la complémentarité essentielle de leurs activités pour s'unir dans ce service de la musique qui est leur raison d'être commune.

\section{Marc Honegger}

La musicologie historique ne peut se passer d'analyse, ni celle-ci d'un cadre historique ; la collaboration de ces deux disciplines s'impose donc mais ne saurait s'établir qu'à la condition que soient respectées certaines exigences fondamentales : (1) ne pas oublier que la musique est susceptible de véhiculer d'importantes données extra-musicales ; (2) éviter le jeu gratuit d'une analyse qui ne tiendrait pas compte de la fonction de l'œuvre ou de son sens ; (3) faire la part de l'essentiel et de l'accessoire ; (4) ne pas faire abstraction des données historiques mais replacer l'œuvre dans son cadre en évitant tout amalgame de chronologie ou d'esthétique ; (5) se garder de privilégier des œuvres comportant une forte proportion d'éléments codés ; et (6) se méfier de la notion de progrès en art et ne faire d'aucun système théorique le garant de l'intérêt ou de l'actualité d'une œuvre.

\section{Jean-Jacques Nattiez}

Le texte rappelle les raisons épistémologiques et institutionnelles qui expliquent la séparation entre les deux tendances et ensuite s'interroge sur la validité de l'opposition entre diachronie et synchronie, en précisant que la description des formes, desstructures et des styles justifie l'autonomie synchronique. Il cherche 
ensuite à montrer qu'une autonomie du diachronique est également justifiée. C'est à partir du moment où on veut passer de la description immanente à l'explication historique et, de façon plus générale, poïétique, que la question de mise en rapport des deux types de discours se pose. On peut caractériser leur différence en opposant le « pigisme » du discours historique et le systématisme des approches immanentes. Enfin, on peut définir le point de contact possible entre musicologie historique et music theory comme la recherche de la pertinence poïétique paramètre par paramètre selon un aller-retour entre analyse immanente et poïétique.

\section{Feminist Theory and Music II: A Continuing Dialogue Eastman School of Music, Rochester, New York 17-20 June 1993}

\section{Virginia Caputo and Roberta Lamb}

Feminist Theory and Music II did advance the dialogues begun at the first such conference held two years ago, and, it seems, the dialogue will continue. Before we left Eastman, the University of California at Riverside was named as the location for the third (1995) conference. Feminist Theory II was much larger, of very high quality, no less provocative, and included more Canadians than the original conference. Five music education-related presentations - a majority within the conference - were Canadian, along with one theory paper and five musicology papers. In fact, so many Canadians registered for the conference that we spontaneously arranged a 'Canadian Caucus' dinner attended by about twenty!

The conference was organized around concurrent sessions scheduled over three days which presented the usual dilemma to those who did not want to miss anything. Lecture/recitals and 'composers on their works' were scheduled for late afternoons. Study sessions, as well as regular sessions, and concerts filled the evenings. Approaches and topics were varied, diverse, and controversial. Broader issues addressed included questions of theory, analysis, practice, patronage, theories of reception, 'voice,' the body, race, and sexualities. Musical examples ran the gamut from k.d. lang to Giovanni Sances, Billie Holiday to Claude Debussy, Clara Schumann to Libby Larsen, and Taarab to Prespa Albanians.

The conference plenary address, "Feminist Queries about Theory and Analysis," was an SMT reprise, updated and (according to the panel) presented to a much more receptive audience. Versions of most of these papers by Fred Maus, Marion Guck, Susan McClary, Marianne Kielian-Gilbert and Suzanne 